\title{
Search for Very High Energy Candidate Sources Among the Fermi Sources
}

\author{
Brian van Soelen ${ }^{* \dagger}$ \\ University of the Free State, Bloemfontein, South Africa \\ E-mail: vansoelenbduts.ac.za
}

\section{Lizelke Klindt \\ University of the Free State, Bloemfontein, South Africa}

\section{P.J. Meintjes}

University of the Free State, Bloemfontein, South Africa

\section{Böttcher}

North-West University, Potchefstroom, South Africa

\section{P. Väisänen}

South African Astronomical Observatory, Cape Town, South Africa

\section{Hanlon}

University College Dublin, Dublin, Ireland

The Fermi gamma-ray telescope has detected a host of new gamma-ray sources since it began operation. While the 2LAC catalogue classifies 1017 extragalactic sources, there is still no clear association with approximately 18 per cent of them. We are undertaking a multi-wavelength campaign to identify the counter parts of these sources and search for potential Very High Energy (VHE) candidates. The project is focussing on southern hemisphere sources and undertaking observations from South African observatories. The early stages of this project have focussed on identifying candidate sources and undertaking optical spectroscopy observations with the Southern African Large Telescope (SALT) and the SAAO 1.9-m telescope located at the Southern African Astronomical Observatory (SAAO). We present an overview of this multi-wavelength project along with recent results obtained.

Frontier Research in Astrophysics

26 - 31 May, 2014

Mondello (Palermo), Italy

\footnotetext{
* Speaker.

${ }^{\dagger}$ This paper uses observations made at the South African Astronomical Observatory (SAAO)
} 


\section{Introduction}

Active Galactic Nuclei (AGN) present some of the most powerful sources in the universe, with some systems detected from radio up to Very High Energy gamma-ray energies. The unified model for AGN is that they are driven by Supermassive Black Holes at the centre of galaxies, accreting material from the surrounding region. The systems can also produce relativistic jets which emit non-thermal radiation. The observational differences between AGN are, in part, the result of our viewing angle of the systems and the direction of the bulk flow of the jet [四. For example, emission from systems that are observed along lines-of-sight that lie close to, or along the flow of the jet, is highly Doppler boosted, greatly enhancing the observed luminosity of the system - such systems are collectively classified as blazars.

The Spectral Energy Distribution (SED) of blazars is clearly divided into two components: a lower energy component extending from radio up to X-ray, and a second component at high energies. The lower-energy component is produced through synchrotron emission, while both leptonic and hadronic processes have been argued for the high energy component of the SED. Both models are able to well reproduce the SED. In addition, blazars can be subdivided into Low, Intermediate, and High Synchrotron peaked, based on the observed peak in the Synchrotron component. See, for example, [l] and references therein for a more detailed discussion.

The Fermi Second LAT AGN Catalogue (2LAC) [3] presented 1017 gamma-ray sources ( $|b|>$ $10^{\circ}$ ) which were categorized as AGN and associated with counterparts through three statistical association tests. A further "clean sample" of a subset of 886 sources was also assigned which did not suffer from analysis issues and had gamma-ray sources that were only associated with one counterpart through the statistical tests. Of these, $157(\sim 17 \%)$ were classified as "AGN of unknown type" (AGU). We are beginning a processes of undertaking multi-wavelength observations of a number of these sources in order to attempt to establish redshifts, to better establish their counterpart association, and to characterize the systems. This project has initially focused on systems which are observable from South African observatories as constrained by the sources' declination and optical/radio brightness.

In addition, we are particularly interested in searching for systems which are good candidates for Very High Energy (VHE) observations. Promising VHE candidate sources should, in general, have low redshifts (since VHE gamma-rays are attenuated by the extragalactic background light, e.g. [四]), exhibit gamma-ray variability, and present hard gamma-ray photo indices. It is for this reason that the initial stages of the project has thus focused on determining the redshift of the AGU sources.

Below we discuss the progress of this project thus far, briefly discussing the multi-wavelength observatories available in South Africa, the selection criteria, planned and on-going observations, as well as initial results obtained.

\section{South African Observatories}

Though the main focus of South African astronomy has, historically, been optical astronomy, radio observations have been undertaken at the Hartebeesthoek Radio Astronomy Observatory (HartRAO) since the 1970s. South Africa is making huge strides in radio astronomy with 
the development of KAT-7/MeerKAT and the planned construction of the Square Kilometre Array (SKA). Below, we briefly outline some of the optical and radio telescopes which are currently available, and those that will be constructed in the near future.

\subsection{Optical Observatories}

\subsubsection{South African Astronomical Observatory}

The telescope site of the South African Astronomical Observatory (SAAO) is located near Sutherland in the Northern Cape, South Africa. The site hosts the Southern African Large Telescope (SALT) as well as a number of smaller SAAO and guest telescopes. ${ }^{1}$

SALT [5] is a 10-m, prime focus optical telescope based on the design of the Hobby-Eberly Telescope. The primary mirror, which consists of 91 segments, is at a fixed elevation of $53^{\circ}$. The telescope hosts four instruments, namely: SALTICAM, a science imaging camera operating between 3200-9000 ^ [ [] , the RSS (Robert Stobie Spectrograph) a low-to medium resolution spectrograph [ $[\mathbb{Z}]$, the BVIT (Berkeley Visible Image Tube) a high-time resolution photometer [ $[\mathrm{d}]$, and the HRS (High Resolution Spectrograph). The most recent instrument, the HRS, started science observations in 2014. Observations with SALT will focus on obtaining broad wavelength spectra to search for and identify spectral features and lines.

In addition to SALT, brighter targets will also be observed with the SAAO 1.9-m telescope. Both spectroscopy and high speed photometry are planned using the "grating spectrograph" and SHOC (Sutherland High Speed Optical Cameras) respectively.

\subsubsection{Boyden Observatory}

The Boyden observatory, located approximately $30 \mathrm{~km}$ outside of Bloemfontein South Africa, is run by the Department of Physics, University of the Free State (UFS) and hosts the UFS/Boyden 1.5m optical telescope and the Watcher Robotic Telescope (40 $\mathrm{cm}$ Cassegrain design) operated by the University College of Dublin.

The UFS/Boyden 1.5-m telescope is equipped with an Apogee U55 CCD camera, used for photometric observations. The Watcher Robotic Telescope is equipped with a Andor EMCCD, also for photometric observations. The Watcher Robotic Telescope is part of the GLORIA project ${ }^{2}$ and focuses on follow-up optical photometry of gamma-ray bursts, though free time can and has been used for monitoring of AGN sources (see e.g. []).

\subsection{Radio Observatories}

\subsubsection{Hartebeesthoek Radio Astronomy Observatory}

The main research telescope at HartRAO, which is located north-west of Johannesburg, South Africa, is the HartRAO 26-m telescope, which operates at 1.3, 2.5, 3.5, 6, 13 and $18 \mathrm{~cm}$. In addition to its participation in the VLBI, the telescope is also used for single dish observations of AGN (see e.g. [ए]] for a discussion of a radio monitoring campaign of unidentified EGRET sources, a precursor project to the one discussed here, undertaken with HartRAO). Single dish observations

\footnotetext{
${ }^{1}$ Further information on SALT and SAAO can be found at http://astronomers.salt.ac.za/ and http://www.saao.ac.za/.

${ }^{2} \mathrm{http}: / /$ gloria-project.eu
} 
are undertaken by making "drift scans" of the target, and have been used to undertake monitoring of other Fermi AGN sources (see e.g. [प]]).

\subsubsection{KAT-7, MeerKAT and SKA}

The development of radio astronomy is rapidly growing in South Africa with the decision that a large portion of the SKA telescope will be located in South Africa. The planning and bid for SKA has lead to the development of the 7-dish test array KAT-7 (KAT: Karoo Array Telescope) located in the Northern-Cape, South Africa, and the planned 64 dish MeerKAT array is currently under construction. MeerKAT will eventually become part of the first phase of SKA [[2]].

\subsection{Gamma-ray observatories}

While not in South Africa, Nambia (on the north-east boarder of South Africa), hosts the H.E.S.S. (High Energy Stereoscopic System) gamma-ray telescope, and is one of two candidate locations of the southern station of the CTA (Cherenkov Telescope Array). This provides an important opportunity for South Africa to participate in multi-wavelength and High Energy astrophysics. South Africa is well suited to provided multi-wavelength support for IACTs (Imaging Air Cherenkov Telescopes) situated in Namibia.

\section{Selection of AGU}

The selection criteria, based on the previous study by [ए]], used to selected targets from 2LAC for this study are (see [[13] for a more detailed discussion):

1. Selected candidates must have no redshift measurement. This has also been checked with newer publications published after the release of 2LAC (e.g. [ए4]]).

2. Select only candidates above $|b|>10^{\circ}$ to exclude sources that lie within the galactic plane. This criteria is already used within the $2 \mathrm{LAC}$

3. Only radio bright sources, $>100 \mathrm{mJy}$ at $4.85 \mathrm{GHz}$ (as catalogued in [15]]), were selected to allow the sources to be observable with the HartRAO 26-m telescope

4. Selected sources must lie within the observable declination limits of the South African telescopes; a limit of $\delta<+20^{\circ}$ was used

5. Where potential optical counterparts are available, selected sources must be brighter than 20th magnitude

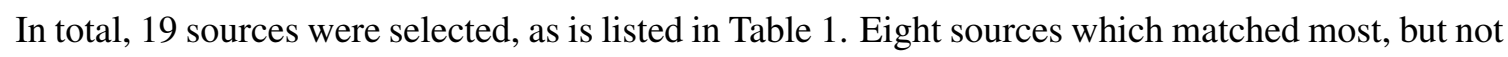
all, selection criteria were also included in the study (highlighted in bold in the table). For example, 2FGL J0730.6-6607 was still included because, while the proposed radio counterpart is fainter than our criteria, the systems displaces a hard photon index $(\Gamma=1.34)$ making it a promising candidate for possible VHE emission. 
Table 1: Nineteen blazar candidates selected among the unidentified Fermi 2LAC objects [[ए3]. Those highlighted in bold match most, but not all, of the selection criteria.

\begin{tabular}{|c|c|c|c|c|}
\hline 2LAC name & Radio counterpart & $\begin{array}{c}\mathrm{V} \\
(\mathrm{mag})\end{array}$ & $\Gamma$ & $\begin{array}{c}\text { Radio Flux } \\
\text { (at } 4.85 \mathrm{GHz}) \\
(\mathrm{mJy})\end{array}$ \\
\hline 2FGL J0044.7-3702 & PKS J0045-3705 & 19.6 & 2.57 & 330 \\
\hline 2FGL J0201.5-6626 & PMN J0201-6638 & 20.56 & 2.25 & 168 \\
\hline 2FGL J0644.2-6713 & PKS 0644-671 & 20.69 & 2.16 & 218 \\
\hline 2FGL J0730.6-6607 & CRATES J073047-660226 & 15.13 & 1.34 & 82 \\
\hline 2FGL J0855.1-0712 & $3 C 209$ & 19.78 & 2.62 & 1157 \\
\hline 2FGL J0919.3-2203 & NVSS J091922-220757 & 19.95 & 2.00 & 26 \\
\hline 2FGL J1059.0+0222 & PMN J1058+0225 & - & 2.29 & 97 \\
\hline 2FGL J1106.3-3643 & PMN J1106-3647 & 19.4 & 2.2 & 53 \\
\hline 2FGL J1154.1-3242 & PKS 1151-324 & 18.88 & 2.03 & 212 \\
\hline 2FGL J1218.8-4827 & CRATES J121901-482624 & 17.53 & 2.4 & 65 \\
\hline 2FGL J1407.5-4257 & PKS 1404-427 & 17.47 & 1.91 & 149 \\
\hline 2FGL J1617.6-2526 & PMN J1617-2537 & - & 2.52 & 120 \\
\hline 2FGL J1624.4+1123 & MG1 J162441+1111 & 17.64 & 2.65 & 113 \\
\hline 2FGL J1803.6+2523 & NVSS J180312-252118 & 14.19 & 2.83 & 166 \\
\hline 2FGL J1955.0-5639 & 1RXS J195503.1-564031 & 17.25 & 1.88 & 9 \\
\hline 2FGL J2040.2-7109 & PKS 2035-714 & 17.47 & 2.03 & 481 \\
\hline 2FGL J2049.8+1001 & PKS 2047+098 & - & 2.38 & 295 \\
\hline 2FGL J2108.6-1603 & NVSS J210833-160724 & - & 2.59 & 7 \\
\hline 2FGL J1848.6+3241 & IVS B1846+326 & 17.77 & 2.43 & 1015 \\
\hline
\end{tabular}

\section{Ongoing observations}

\subsection{SAAO 1.9-m}

Initial spectroscopic observations of the listed targets were undertaken with the SAAO 1.9-m telescope between 2014 May 21 - June 3. The grating spectrograph was used (with grating 7) to obtain a broad wavelength coverage (wavelength range of $4200 \AA$, wavelength resolution $5 \AA$ ) to search for potential spectral lines and features. Multiple pointings were undertaken for each source to obtain a higher signal to noise ratio. The typical integration time of each pointing was $\sim 1$ hour. Initial results have been obtained for seven sources, showing potential Ca II H\&K lines. Preliminary analysis places these sources within a redshift range of $0.11<z<0.37$ (see [ए]] for a more detailed discussion). For example, the preliminary spectra obtained for 2FGL J0730.6-6607 shows potential $\mathrm{Ca}$ II H\&K lines at $\lambda 4388$ and $\lambda 4351$ with a Ca break value of less than $40 \%$ and a redshift of $z \approx 0.11$ (Fig. 四) The presence of the Ca depression at $4000 \AA$ is a signature of thermal emission in the intergalactic gas, and therefore a shallow Ca depression indicates the presence of additional non-thermal emission which, for example, occurs within the jet structure of blazars. The depression has a break value $K_{4000}<40 \%$ for blazars, which can therefore be used to differentiate 
between blazars (FSRQs and BL Lacs) and normal radio galaxies (e.g. [ए7, [8, प0]). From these preliminary results it can be concluded that strong non-thermal emission is present in this target and is therefore a potential blazar object. Further observations with SALT will be used to confirm this result.

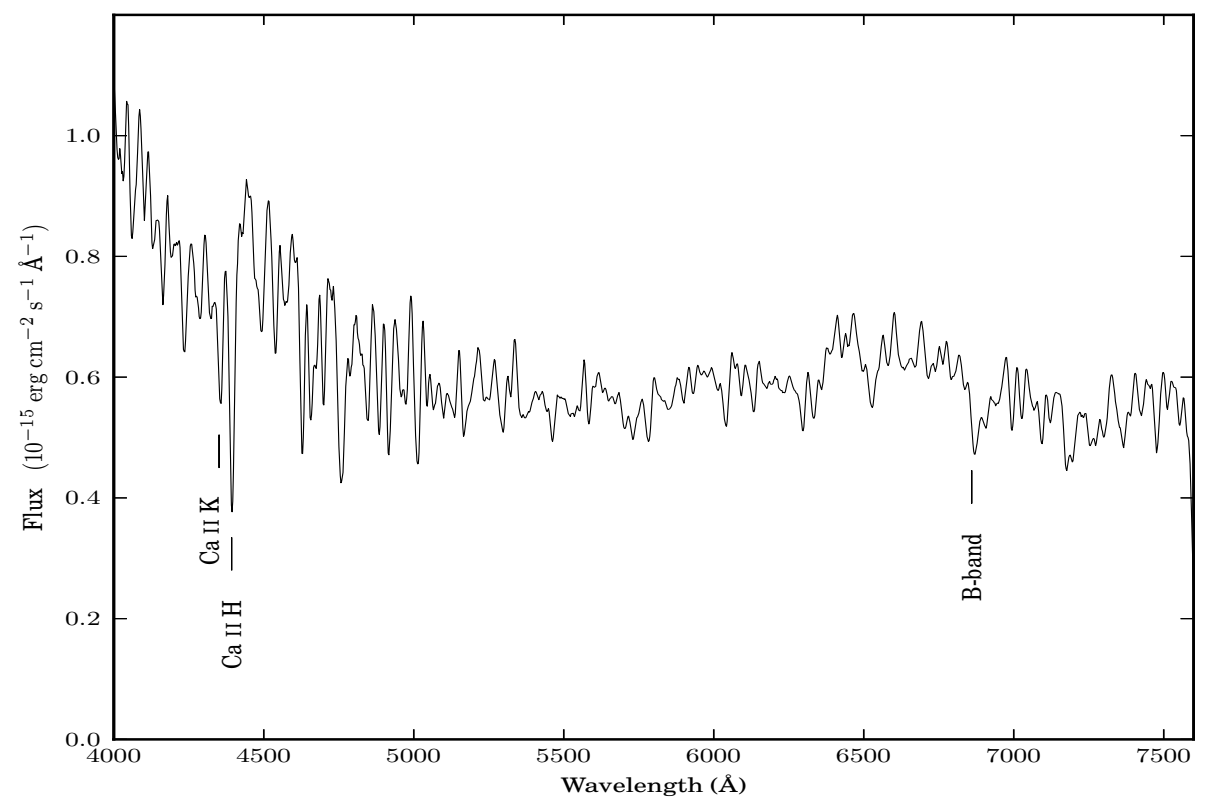

Figure 1: One example of spectroscopic observations of a AGU undertaken with the SAAO 1.9-m telescope - 2FGL J0730.6-6607. Potential Ca II H\&K lines are present at $\lambda 4388$ and $\lambda 4351$.

\subsection{Watcher Robotic Telescope}

The Watcher Robotic Telescope has been involved with some on-going AGN monitoring [Q $⿴$ ]. It is intended that additional time (not used for gamma-ray burst observations) will be used to for monitoring of AGN sources. A technical fault with the Watcher Robotic Telescope led to a failure of the automatic weather system, allowing the dome to remain open during a thunderstorm during 2013 December. This led to the system being off-line during the first half of 2014. The system has since been repaired (July 2014) and is fully operational.

\section{Future work}

Further observations of the remaining candidate sources, as well as additional follow-up observations, are planned at optical as well as at radio frequencies. Photometric observations will be undertaken with the SAAO 1.9-m using SHOC during December 2014, to search for potential (rapid) variability of the sources. Furthermore, time has been obtained on SALT to undertake broadband spectroscopic follow-ups of five sources. Radio observations with the HartRAO 26-m telescope are also planned for early 2015.

In addition, further multi-wavelength observations (in particular at UV and X-ray frequencies) are required to establish the peak in the synchrotron emission, to search for High Synchrotron 
Peaked sources. This may further allow for a measurement of the accretion disk emission and a determination of the mass of the black hole in the system (e.g. [미, [20]). This will be considered through available archival data as well as possible Swift observation proposals for promising candidates.

\section{Discussion and Conclusion}

As approximately 18 per cent of the Fermi $2 \mathrm{LAC}$ catalogue sources are still given as "unclassified", we are undertaking a multi-wavelength campaign to identify and classify the counterparts of some of these sources. We are focussing on southern hemisphere sources which are observable from South African institutes. Here we have presented the early results from this project, presenting the selected candidate sources and discussing the observations that are currently ongoing or planned. Initial results have already been able to establish redshift estimates for a few of the sources.

Through this classification of Fermi-LAT systems, we are searching for potential VHE energy sources which may be observable with current (or future) IACT. Here the results of the recent observational campaign are discussed. The establishment of the redshift is an important property as attenuation by the extragalactic background light will prevent observations at VHE, and it is therefore necessary to identify nearby sources.

Acknowledgments BvS and LK acknowledges that this work is based on the research supported in part by the National Research Foundation of South Africa. LH acknowledges support from Science Foundation Ireland (grant 07/RFP/PHYF295) and the EU FP7 (grant agreement no. 283783). This paper uses observations made at the South African Astronomical Observatory (SAAO).

\section{References}

[1] C.M. Urry, P. Padovani, Unified Schemes for Radio-Loud Active Galactic Nuclei, PASP 107 (1995) 803

[2] M. Böttcher, Modeling the Spectral Energy Distributions and Variability of Blazars (2012) - eConf C1111101

[3] M. Ackermann et al. The Second Catalog of Active Galactic Nuclei Detected by the Fermi Large Area Telescope, ApJ 742 (2011) 171

[4] R.J. Gould, G.P. Schréder, Opacity of the Universe to High-Energy Photons, Phys. Rev. 155 (1967) 1408

[5] D.A.H. Buckley, G.P. Swart, J.G. Meiring, Completion and commissioning of the Southern African Large Telescope, SPIE 6267 (2006) 32

[6] D. O'Donoghue et al., First science with the Southern African Large Telescope: peering at the accreting polar caps of the eclipsing polar SDSS J015543.40+002807.2, MNRAS 372 (2006) 151

[7] E.B. Burgh et al., The Prime Focus Imaging Spectrograph for the Southern African Large Telescope: optical design, SPIE 4841 (2003) 1463 
[8] O.H.W.S. Siegmund et al., High time resolution astronomical observations with the Berkeley Visible Image Tube, AIP Conf. Proc. 984 (2008) 103

[9] P. Tisdall et al. Blazar Monitoring with the Watcher Robotic Telescope, in the proceedings of Third Workshop on Robotic Autonomous Observatories, Revista Mexicana de Astronomia y Astrofisica 45 (2014) 71

[10] P. Nkundabakura, P.J. Meintjes, Unveiling the nature of two unidentified EGRET blazar candidates through spectroscopic observations, MNRAS 427 (2012) 859

[11] P. van Zyl, M. Gaylard, Examining the radio to gamma-ray correlation of the blazar PKS 1424-418 during its flaring state, in the proceedings of High Energy Astrophysics in Southern Africa 2014, (2014)

[12] R.S. Booth., J.L. Jonas, An Overview of the MeerKAT Project, African Skies 16 (2012) 101

[13] L. Klindt, B. van Soelen, P.J. Meintjes,Unidentified Fermi-2LAC sources: identification of candidate sources, in proceedings of High Energy Astrophysics in Southern Africa 2014 (2014)

[14] M.S. Shaw et al. Spectroscopy of the Largest Ever gamma-Ray-selected BL Lac Sample, ApJ 764 (2013) 135

[15] P.C. Gregory et al., The GB6 Catalog of Radio Sources, ApJS 103 (1996) 427

[16] [12] L. Klindt, P.J. Meintjes, B. van Soelen, Multi-wavelength classification of unidentified AGN in the Fermi-2LAC catalogue, in proceedings of SAIP 2014 (2014)

[17] H. Landt, P. Padovani, P. Giommi, The classification of BL Lacertae objects: the Ca H\&K break, MNRAS 336 (2002) 945

[18] E. Galbiati et al. XMM-Newton spectroscopy of an X-ray selected sample of RL AGNs, A\&A 430 (2005) 927

[19] L. Ferrarese, D. Merritt, A Fundamental Relation between Supermassive Black Holes and Their Host Galaxies, ApJ 539 (2000) L9

[20] K. Gebhardt et al., A Relationship between Nuclear Black Hole Mass and Galaxy Velocity Dispersion, ApJ 539 (2000) L13

\section{DISCUSSION}

JAMES BEALL: Very interesting. How soon could we expect results from your observations of the LAT sources?

BRIAN VAN SOELEN: This, like all observational work, will depend on how long it takes to obtain useful spectra and interesting results. I don't want to put a fixed time line on this, but we are already undertaking spectroscopic observations with the SAAO 1.9-m and it will depend on these observations. [Some preliminary results were subsequently included in this proceedings.] 